\title{
Tratamiento
}

\section{ENFERMEDAD DE CHAGAS}

\section{Cuantificación de la viabilidad celular con azul de tiazolina por el método MTT en ensayos de toxicidad in vitro del veneno de ofidios a parásitos Trypanosomatidae}

Alicia Jorquera, Leomery Romero, Rebeca Ramírez, Mayerlin Rivero, Demetrio Kiriakos

Centro de Investigaciones en Ciencias de la Salud, Universidad de Oriente, Núcleo de Anzoátegui, Venezuela

Introducción. La evaluación del efecto antiparasitario de los fármacos emplea convencionalmente el conteo de viabilidad celular con azul de tripano; sin embargo, éste resulta muy laborioso. En este estudio se estandarizó el método espectrofotométrico con MTT (3-[4,5-dimetiltiazol2-yl]-2,5-bromuro de difeniltetrazolium) o azul de tiazolina, para cuantificar la viabilidad de Leishmania amazonensis y Trypanosoma cruzi en ensayos de toxicidad del veneno de ofidios.

Materiales y métodos. Para estandarizar el método MTT, se incubaron $900 \mu \mathrm{l}$ de cultivos de concentración entre 0 y $3,5 \times 10^{6}$ parásitos/ $\mathrm{ml}$ (epimastigotes de $T$. cruzi o promastigotes de L. amazonensis) en placas de 24 pozos con 100 $\mu$ de MTT $(5 \mathrm{mg} / \mathrm{ml})$ durante 18 horas $/ 28^{\circ} \mathrm{C}$. La reacción se paró con 10 \% SDS-HCl (1M) y se leyó la absorbancia (DO a $595 \mathrm{~nm}$ ). Para los ensayos de toxicidad se empleó veneno de Bothrops colombiensis y Crotalus durissus cumanesis en concentraciones entre 0 y $40 \mu \mathrm{g} / \mathrm{ml}$.

Resultados. La relación entre la DO resultante de los cristales púrpura-morado característicos de la metabolización del MTT por los parásitos vivos y la concentración de parásitos originó valores de $r=0,999$ ( $L$. amazonensis) y $r=0,995$ ( $T$. cruzi) en el análisis de regresión lineal, lo que demuestra la validez del método para cuantificar la viabilidad celular. Mediante las curvas de crecimiento se verificó el efecto tóxico dependiente de la dosis del veneno sobre los parásitos, cuantificando la viabilidad con MTT y con azul tripano.

Conclusiones. Se demuestra la precisión y facilidad de uso del MTT en ensayos de toxicidad del veneno de ofidios sobre los parásitos. Al presente se realizan los ensayos comparativos para calcular la ID50 de cada veneno con el uso del MTT.

\section{Evaluación in vitro de diaminas y aminoalcoholes de cadena larga frente a epimastigotes de Trypanosoma cruzi \\ Ana L. Legarda-Ceballos ${ }^{1}$, Esther del $\mathrm{Olmo}^{2}$, Ricardo Escarcena², Luis A. Bustos², Antonio Muro', Juan C. Dib $^{3}$, Arturo San Feliciano ${ }^{2}$ \\ 1 Inmunología Parasitaria y Molecular, Centro de Investigación de Enfermedades Tropicales, Universidad de Salamanca, Salamanca, España \\ 2 Departamento de Química Farmacéutica, Facultad de Farmacia, Universidad de Salamanca, Salamanca, España \\ 3 Fundación Salud para el Trópico, Santa Marta, Colombia}

Introducción. La enfermedad de Chagas continúa siendo un problema importante de salud pública en América Latina. Los medicamentos disponibles, nifurtimoxy benznidazol, noproducen mejoríaclínica satisfactoria en la fase crónica de la enfermedad y provocan numerosos efectos adversos, lo que determina la necesidad de descubrir nuevos y mejores agentes terapéuticos. Ello ha motivado la evaluación in vitro de otros compuestos frente a epimastigotes de Trypanosoma cruzi.

Materialesy métodos.Seevaluaron 25 compuestos sintéticos nuevos a seis concentraciones (20 a $0,1 \mu \mathrm{g} / \mathrm{ml}$ ), sobre las cepas de origen colombiano MG (MHOM/CO/04/MG) y JEM (MHOM/CO/05/ JEM), obtenidas de pacientes en fase aguda $y$ pertenecientes al grupo I y haplotipo la, mediante tinción vital con azul tripán y lectura microscópica. La citotoxicidad de los compuestos activos se evaluó sobre macrófagos $\mathrm{J774}$ de ratón. Como controles de referencia se utilizaron nifurtimox y benznidazol.

Resultados. Frente a la cepa MG (JEM), 6 (3) compuestos entre los evaluados resultaron más potentes que nifurtimox y otros 5 (8) mostraron valores de $\mathrm{IC}_{50} / \mathrm{IC}_{90}$ del mismo orden que el fármaco de referencia. El mejor compuesto resultó 4,5 veces más potente que nifurtimox y 4,9 veces mejor que benznidazol. El índice de selectividad parásito/ macrófago alcanzó niveles superiores a 20.

Conclusiones. Tanto los derivados de diaminas como de aminoalcoholes de cadena larga se constituyen en nuevos tipos de compuestos potencialmente útiles frente a $T$. cruzi en comparación con los 
fármacos en uso, y algunos de ellos serán evaluados frente a amastigotes, para progresar, en su caso, hacia ensayos preclínicos.

Financiación. ISCIII-España: RICET (RD06/0021/ 0022) y FIS (PI 060782) y Centro Investigación de Enfermedades Tropicales de la Universidad de Salamanca (CIETUS).

\section{- $\bullet$}

\section{Evaluación in vitro de compuestos heterocíclicos fusionados frente a epimastigotes de Trypanosoma cruzi}

Ana L. Legarda-Ceballos ${ }^{1}$, Esther del Olmo², Ricardo Escarcena ${ }^{2}$, Sobinson Mananandro², Antonio Muro ${ }^{1}$, Juan C. Dib ${ }^{3}$, Arturo San Feliciano ${ }^{2}$

1 Inmunología Parasitaria y Molecular, Centro de Investigación de Enfermedades Tropicales, Universidad de Salamanca, Salamanca, España

2 Departamento de Química Farmacéutica, Facultad de Farmacia, Universidad de Salamanca, Salamanca, España

3 Fundación Salud para el Trópico, Santa Marta, Colombia

Introducción. La enfermedad de Chagas aún representa un problema importante de salud pública en América Latina. Los medicamentos disponibles, nifurtimox y benznidazol, no producen respuesta clínica satisfactoria en la enfermedad crónica y provocannumerososefectosadversos, determinando la necesidad de descubrir nuevos y mejores agentes terapéuticos. Ello ha motivado la evaluación in vitro de una serie de imidazoisoindoles, que ya habían mostrado actividad frente a Plasmodium, ahora frente a epimastigotes de Trypanosoma cruzi.

Materiales y métodos. Se evaluaron 29 compuestos sintetizados en nuestros laboratorios a seis concentraciones $(20-0,1 \mu \mathrm{g} / \mathrm{ml})$, sobre las cepas de origen colombiano MG (MHOM/CO/04/ MG) y JEM (MHOM/CO/05/JEM), obtenidas de pacientes en fase aguda y pertenecientes al grupo I y haplotipo la, mediante la técnica colorimétrica XTT. La citotoxicidad de los compuestos activos se evaluó sobre macrófagos $\mathrm{J774}$ de ratón. Como control de referencia se utilizó benznidazol.

Resultados. Frente a la cepa MG (JEM), 5 compuestos entre los evaluados resultaron más potentes que benznidazol y otros 4 presentaron valores de $I_{50} / I_{90}$ del mismo orden que el fármaco de referencia. El mejor compuesto resulto 68 veces más potente que el benznidazol. El índice de selectividad parásito/macrófago alcanzó, en el mejor caso, niveles superiores a 140 .

Conclusiones. El sistema heterocíclico de imidazo[2,1-a]isoindolol constituye una buena base estructural para la preparación de compuestos potencialmente útiles frente a $T$. cruzi. Algunos compuestos superaron con claridad la actividad tripanocida del fármaco de referencia, manteniendo niveles de citotoxicidad bastante menores. Consecuentemente, varios compuestos de la serie se han seleccionado para efectuar nuevas evaluaciones tendentes a justificar posteriores ensayos preclínicos.

Financiación. ISCIII-España: RICET (RD06/0021/ 0022) y FIS (PI 060782) y Centro Investigación de Enfermedades Tropicales de la Universidad de Salamanca (CIETUS).

\section{Sensibilidad in vitro a nifurtimox y benznidazol de aislamientos de Trypanosoma cruzi provenientes de transmisión oral y vectorial en Venezuela}

Arturo Muñoz, Attilio Santaniello, Alexis Pereira, Josmairy Yannuzzi, Zoraida Díaz-Bello, Belkisyolé Alarcón de Noya

Sección de Inmunología, Instituto de Medicina Tropical, Universidad Central de Venezuela, Caracas, Venezuela

Introducción. Los frecuentes reportes de brotes agudos de enfermedad de Chagas por la ingestión de comida contaminada, ilustran la importancia de este modelo de transmisión. Venezuela no ha escapado a esta realidad y hasta el presente han ocurrido tres brotes significativos con una tasa de cura inferior a la reportada en la bibliografía.

El presente estudio propone evaluar, por primera vez, la sensibilidad in vitro de aislamientos parasitarios provenientes del brote agudo de Chagas por transmisión oral, ocurrido en Caracas, Venezuela, en el 2007, a los fármacos nifurtimox y benznidazol.

Materiales y métodos. Se estudiaron cuatro aislamientos venezolanos, dos por transmisión oral y dos por transmisión vectorial. En una placa de 96 pozos se colocaron $1 \times 10^{9}$ tripomastigotes $/ \mathrm{ml}$ y se incubaron por 72 horas con los fármacos $(0,01$ $1.000 \mu \mathrm{g} / \mathrm{ml}$ ). La actividad de los fármacos fue determinada por el ensayo colorimétrico del MTT. Los resultados se expresaron en concentraciones inhibitorias $50\left(\mathrm{Cl}_{50}\right)$. La significancia estadística se determinó con una prueba $\mathrm{T}$ de Student. Los valores de $p<0,05$ se considerados significantes.

Resultados. Para el benznidazol, se observaron rangos similares en la actividad del fármaco entre los aislamientos de transmisión vectorial $\left(\mathrm{Cl}_{50}>3.800\right.$ $\mu \mathrm{M})$ y los aislamientos de transmisión oral $\left(\mathrm{Cl}_{50}\right.$ $650,53$ a $687,03 \mu \mathrm{M})$; todos los aislamientos 
fueron más sensibles al fármaco nifurtimox $\left(\mathrm{Cl}_{50}\right.$ $60,84$ a 213,96 $\mu \mathrm{M})$; se encontró una diferencia estadísticamente significativa $(p<0,05)$ en cuanto a la actividad de los fármacos sobre los aislamientos provenientes de la transmisión oral con respecto a los de transmisión vectorial.

Conclusión. Los resultados mostraron algunas diferencias en la actividad de los fármacos nifurtimox y benznidazol sobre los aislamientos de Trypanosoma cruzi estudiados, y los mismos serán considerados para estudios posteriores in vivo.

$$
\bullet \bullet
$$

\section{Efecto de la administración del etanercept sobre la evolución de la enfermedad aguda de Chagas en ratones infectados experimentalmente}

Héctor Rodríguez-Angulo ${ }^{1}$, Endher Castillo², Edward

Cárdenas ${ }^{2}$, Alfredo Mijares ${ }^{1}$, Enrique Vásquez ${ }^{3}$

1 Laboratorio de Fisiología de Parásitos, Instituto Venezolano de Investigaciones Científicas, Venezuela

2 Facultad de Ciencias de la Salud, Universidad Centroccidental Lisandro Alvarado, Barquisimeto, Venezuela

${ }^{3}$ Laboratorio de Neurofisiología, Instituto Venezolano de Investigaciones Científicas, Venezuela

Introducción. El factor de necrosis tumoral (FNT) se ha asociado a la inflamación durante la enfermedad aguda de Chagas. El conocimiento sobre los efectos de fármacos que bloquean al FNT durante la fase aguda y crónica de la enfermedad es controversial.

En este sentido, el objetivo del trabajo es describir el efecto de la administración del etanercept (Enbrel®) sobre la parasitemia, la algesia mecánica, la motilidad y la curva de mortalidad en ratones con enfermedad de Chagas en fase aguda.

Materiales y métodos. Se infectaron ratones NMRI de $30 \mathrm{~g}$ con 1.000 tripomastigotes $/ \mathrm{g}$ de ratón del aislamiento "potrerito" de Trypanosoma cruzi. El etanercept se administró por vía subcutánea en dosis de $0,83 \mathrm{mg} / \mathrm{kg}$ diarios, los días 1,7 y 7 , con refuerzo el día 14 después de la infección ( $n=8,9$ y 11 , respectivamente, más 21 de grupo sin tratamiento). Se determinó semanalmente la cantidad de parásitos en líquido ascítico, y se cuantificaron la mortalidad y la motilidad horizontal y vertical durante tres minutos en una caja de actividad.

Resultados. En los grupos 7 y $7+14$, se observó un incremento en la tasa de parasitemia a partir de la segunda semana después de la infección con respecto al grupo control y al día 1. En los grupos 7 y $7+14$, el tratamiento revirtió la hiperalgesia y la disminución de la motilidad. Finalmente, hubo un incremento en la tasa de supervivencia en los grupos 7 y $7+14$ con respecto a los controles y al día 1.

Conclusiones. El tratamiento con etanercept revierte el dolor neuropático y el sickness behaviour asociado a la fase aguda de enfermedad de Chagas, e incrementa la supervivencia. Estos resultados sugieren una asociación del FNT con el curso agudo de la enfermedad de Chagas.

$$
\text { - }
$$

\section{Evaluación del efecto de compuestos monosustituidos y disustituidos, acoplados a ergosterona, sobre epimastigotes de Trypanosoma cruzi}

Irina Arocha1 ${ }^{1}$, Sabrina Marsiccobetre ${ }^{1}$, William Colina², Masahisa Hasegawa², Néstor L. Uzcátegui ${ }^{1,2}$, Katherine Figarella $^{1}$

1 Fundación Instituto de Estudios Avanzados, Venezuela

2 Universidad Central de Venezuela, Caracas, Venezuela

Introducción. La enfermedad de Chagas representa un punto focal en investigación, debido a su importante morbimortalidad en el continente americano. Actualmente, la quimioterapia resulta ineficaz en un número importante de casos; además, la inespecificidad de los fármacos ocasiona efectos secundarios adversos que en ocasiones no permite su uso.

Con el objetivo de hallar alternativas terapéuticas que modifiquen el panorama actual de la enfermedad de Chagas, se evaluó el efecto de compuestos acoplados a ergosterona, sobre el parásito Trypanosoma cruzi.

Materiales y métodos. Se trataron cultivos de epimastigotes de T. cruzicon compuestos acoplados a ergosterona y se determinó la concentración inhibitoria 50 para estimar su toxicidad. Se seleccionaron los compuestos con mayor actividad contra el parásito y, posteriormente, se evalúo la morfología por coloración de Giemsa. Además, se analizaron, mediante citometría de flujo, el ciclo celular y los marcadores de muerte celular programada (integridad de membrana plasmática, potencial de membrana mitocondrial, exposición de fosfatidilserina y degradación de ADN). La detección de fragmentación de ADN se hizo por el test TUNEL, mediante microscopía confocal.

Resultados. Los compuestos Erg-GTr y Erg-GTr presentaron $\mathrm{IC}_{50}$ de $3,79 \pm 1,12$ uM y $1,33 \pm 0,61$ $\mathrm{uM}$, respectivamente. Se encontraron cambios morfológicos, como reducción del contenido citoplasmático, forma redonda de las células y 
presencia de dos o más flagelos. Los epimastigotes tratados mostraron un arresto en fase $\mathrm{G}_{2}$ de su ciclo celular. Se detectó ruptura de ADN y reducción del potencial de membrana mitocondrial sin pérdida de la integridad de la membrana plasmática. No se observó exposición de fosfatidilserina.

Conclusiones. Los compuestos azólicos monosustituidos y disustituidos acoplados a ergosterona ejercen una acción citotóxica sobre epimastigotes de T. cruzi. Los resultados obtenidos nos permitieron descartar la necrosis celular, como vía de muerte, y nos orientan hacia procesos de muerte celular programada.

$$
\bullet \bullet
$$

\section{Cationes lipofílicos derivados del ácido gálico: efecto antitripanocida in vitro}

José Jara, Rodrigo López-Muñoz, Antonio Morello, Juan Diego Maya, Jorge Ferreira

Programa de Farmacología Molecular y Clínica, Facultad de Medicina, Universidad de Chile, Santiago, Chile

Introducción. La enfermedad de Chagas es producida por el protozoo Trypanosoma cruzi. El ácido gálico tiene actividad antitripanocida in vitro. En este estudio se ensayaron cationes lipofílicos derivados del ácido gálico que poseen un grupo trifenilfosfonio, y que están conectados al ácido gálico con una cadena alquílica de diferente largo. Se utilizaron compuestos con una cadena de 8,10 , 11 y 12 carbonos (C8, C10, C11 y C12).

Materiales y métodos. Se cultivaron tripomastigotes de T. cruzi (cepa Y) en células VERO. La viabilidad de tripomastigotes y células VERO se determinó mediante reducción de sales de tetrazolio (MTT); las células infectadas se cuantificaron por microscopía directa, asociada a fluorescencia, utilizando la tinción de núcleos con DAPI para identificar los amastigotes intracelulares.

Resultados. Respecto a la viabilidad celular, estos compuestos mostraron un $\mathrm{IC}_{50}$ de $3,02 \pm$ 0,$5 ; 0,60 \pm 0,1 ; 3,64 \pm 1,0 ;$ y $0,85 \pm 0,2 \mu \mathrm{M}$ en tripomastigotes aislados (para C8, C10, C11 y C12, respectivamente); en células VERO los valores de $\mathrm{IC}_{50}$ fueron al menos 20 veces mayores. Al estudiar células VERO infectadas, se encontró que 0,5 $\mu \mathrm{M}$ de $\mathrm{C} 10$ y $\mathrm{C} 12$ disminuyeron el porcentaje de células infectadas de $17,6 \pm 6,6$ (control) a 10,0 $\pm 8,5$ y 5,4 $\pm 2,1$, para $\mathrm{C} 10$ y $\mathrm{C} 12$, respectivamente. Además, C10, C11 y C12 redujeron significativamente el número de amastigotes por célula infectada (17,7 $\pm 9,4$ control, $1,3 \pm 0,8 ; 8,3 \pm 3,7$ y $2,3 \pm 0,8$, para C10, C11 y C12, respectivamente).
Conclusiones. Los cationes ensayados presentan potente actividad antiparasitaria y selectividad al ser comparados con células de mamífero. Los compuestos de cadena de 10 y 12 carbonos fueron los más potentes en parásitos aislados y células infectadas. Estos resultados in vitro apuntan a la potencial utilidad de estos compuestos in vivo como agentes contra la enfermedad de Chagas.

Financiado por proyectos: FONDECYT -1090075 y ACT-112

\section{Tratamiento de escolares infectados con Trypanosoma cruzi: cumplimiento, seguridad y eficacia del programa en Casanare}

Nadia L. González', Fiorella Bianchi ${ }^{1}$, Zulma Cucunubá2, Marleny Montilla², Carolina Flórez², Fernando Rosas ${ }^{3}$, Héctor Freilij ${ }^{4}$, Nubia Silva ${ }^{5}$, Víctor Saavedra' ${ }^{6}$, Luis Eduardo Castro ${ }^{7}$, Felipe Guhl ${ }^{1}$

1 Centro de Investigaciones en Microbiología y Parasitología Tropical, Universidad de los Andes, Bogotá, D.C., Colombia

2 Grupo de Parasitología, Instituto Nacional de Salud, Bogotá, D.C., Colombia

${ }^{3}$ Electrofisiología, Clínica Abood Shaio, Bogotá, D.C., Colombia

${ }^{4}$ Hospital Ricardo Gutiérrez, Buenos Aires, Argentina

${ }^{5}$ ServinSalud Ltda., Casanare, Colombia

${ }^{6}$ Hospital San Silvestre, Casanare, Colombia

7 Secretaría de Salud de Casanare, Yopal, Colombia

Introducción. El tratamiento de la enfermedad de Chagas en niños y adolescentes previene el desarrollo de cardiomiopatías. A su vez, el cumplimiento del tratamiento determina el éxito del mismo, mejora la calidad de vida del paciente y es una medida económicamente favorable, para el paciente y para los sistemas de salud. Este es el primer estudio de tratamiento etiológico con nifurtimox que se realiza en Colombia.

Materiales y métodos. Se tamizaron 3.050 escolares entre 5 y 18 años, en las escuelas de Nunchía y Yopal. Se diagnosticó la infección por Trypanosoma cruzi por métodos serológicos (IFI, ELISA). Los pacientes con diagnóstico positivo y sus acudientes, fueron convocados a sesiones informativas con expertos en temas relacionados con la enfermedad y el tratamiento. Allí tuvieron la oportunidad de manifestar sus inquietudes y diligenciar el consentimiento informado para ingresar al estudio. Los pacientes recibieron nifurtimox, en dosis de $10 \mathrm{mg} / \mathrm{kg} /$ día durante 60 días, con examen físico y seguimiento de laboratorio clínico a los días 0, 20, 40 y 60. Desde 
el día 60 y cada 6 meses después del tratamiento, hasta el momento, se viene haciendo seguimiento serológico y parasitológico de los pacientes.

Resultados. De los 62 pacientes que iniciaron el tratamiento, el 100 \% lo finalizó, uno lo suspendió durante tres días por aparición de efectos secundarios, lo reanudó y finalizó correctamente el esquema. Los efectos adversos más observados fueron: hiporexia $(27,22 \%)$, cefalea $(16,6 \%)$ y astenia (13\%). Los resultados serológicos hasta el momento han mostrado $21 \%$ de seroconversión y $14 \%$ de disminución de títulos de anticuerpos. El cumplimiento del tratamiento y la asistencia la último control fue de $100 \%$; para el seguimiento de 6 y 12 meses, la asistencia fue de $96,7 \%$ y $87,1 \%$, respectivamente.

Conclusiones. El modelo de trabajo a diferentes niveles (institucional, municipal y nacional), permitió garantizar el cumplimiento de los pacientes del tratamiento; de esta manera, se pudo hacer un seguimiento exhaustivo de su desarrollo, los efectos adversos generados y su manejo. Asimismo, el cumplimiento determina la efectividad del medicamento, ya que se obtienen resultados de seroconversión y disminución de títulos de anticuerpos similares a lo reportado en otros países.

\section{Sensibilidad de líneas celulares a la infección in vitro por Trypanosoma cruzi}

Paola Díaz¹, Carol Mesa¹, Diana Muñoz¹, Iván D. Vélez ${ }^{1}$, Sara Robledo ${ }^{1,2}$

1 Programa de Estudio y Control de Enfermedades Tropicales, Universidad de Antioquia, Medellín, Colombia

2 Facultad de Medicina, Universidad de Antioquia, Medellín, Colombia

Introducción. La tripanosomiasis americana es una enfermedad grave que hasta el momento no tiene una vacuna y los medicamentos existentes son eficaces si se administran al comienzo de la infección, en la etapa aguda; sin embargo, su eficacia disminuye a medida que transcurre más tiempo desde el inicio de la infección. Por lo tanto, se hace necesaria la búsqueda de alternativas terapéuticas. Los sistemas in vitro son de gran utilidad en la evaluación de compuestos con posible actividad tripanocida, y permiten determinar el potencial citotóxico y tripanocida del candidato a medicamento.

Con el fin de identificar el mejor sistema para infecciones in vitro por Trypanosoma cruzi y para evaluar la actividad citotóxica y tripanocida de los compuestos, en el presente trabajo se evaluó la sensibilidad que tienen los tipos de células comúnmente utilizados, a la infección por $T$. cruzi para evaluar in vitro su efectividad tripanocida.

Materiales y métodos. Se infectaron líneas celulares de macrófagos (J774.A1) y de músculo de ratón (C2C12) y macrófagos (U-937) y células humanas epiteliales de cuello uterino (HeLa), con varias concentraciones de parásitos $T$. cruzi, cepa Tulahuen, transfectada con el gen de la $\beta$-galactosidasa, y se determinó el porcentaje de células infectadas por microscopía de luz y por colorimetría, para obtener la $\mathrm{Cl}_{50}$, la cual se calculó por el método Probit.

Resultados. Hasta el momento sólo se tienen resultados de las infecciones con la línea celular U-937, con la cual encontramos una $I_{50}$ de $5,4 \pm 0,5$ por microscopio de luz; además, las diluciones seriadas de los parásitos que expresan $\beta$-galactosidasa mostraron una relación casi lineal entre el número de parásitos y la actividad de la $\beta$-galactosidasa, en un rango de 5000.000 a $80 ` 000.000$ parásitos $/ \mathrm{ml}$, con un $\mathrm{r}^{2}$ de 0,9 .

Conclusiones. Con los resultados que obtengamos esperamos encontrar la mejor línea celulares que nos permita realizar evaluaciones confiables y reproducibles de actividad tripanocida. 